\title{
Relative importance of various stochastic terms and EUV patterning
}

\author{
Patrick Naulleau; Gregg Gallatin \\ Lawrence Berkeley National Lab. \\ Applied Math Solutions, LLC
}

\begin{abstract}
An error propagation stochastic model is described and used to study the impact of both photon and photoresist material sources of line-width roughness (LWR). Based on typical chemically amplified resist parameters, material sources of LWR are shown to be of equal importance to photon sources. Of the material sources, quencher is shown to be the most important input noise term. The results show that it is not the relative quencher noise that ultimately matters but rather the absolute quencher noise relative to the mean produced acid count. The results also show that chemical yield is critical and that benefiting from increased absorptivity also requires the chemical yield to be maintained.
\end{abstract}

1. Introduction

As we approach the atomic scale for patterning, stochastics driven by counting statistics begin to dominate variability concerns. This issue is most commonly discussed from the perspective of photon shot noise but is in fact more generally applicable to also incorporate photoresist material components. Examples of stochastic materials parameters of concern include photoacid generators, quenchers, protecting groups, and generated acids. To predict the statistical limitations of a material process, stochastic resist models $\underline{12.3 .4 .5}$. $-\underline{6}$ are needed as opposed to the traditional mean field models that work only based on average parameters. An example of such a model is the multivariate Poisson propagation model (MPPM).1, 18 . $-\underline{9}$ We summarize the model below and use it to gain insights into the relative importance and implications of various stochastic terms.

2.

Model Description

In the MPPM model, counting statistics theory is used to determine the local nanoscale density of key material, reaction, and exposure components. Figure 1 pictorially shows the flow of the model for the case of a chemically amplified resist.10 The model starts with a given aerial image that represents the infinite time-averaged photon distribution. The first random variable (RV1) represents the absorption process yielding a Poisson distribution of absorbed photons per pixel based on the input dose, pixel volume, and material absorptivity. Both pixel volume (i.e., resist thickness) and absorptivity are considered as deterministic variables. The next step is release of acid that is determined by the acid generation efficiency (or quantum efficiency, RV2) as well as counting statistics of local PAG density (RV3). The acid generation potential at each pixel becomes a sum of Poisson RVs with mean value equal to the quantum efficiency (QE) and the summation count being equal to RV1. The impact of electron blur (a deterministic variable) is incorporated at this point through the implementation of a Gaussian blur of the acid generation potential. The actual generated acid count per pixel is then determined based on the acid generation potential constrained by local saturation effects determined by the local PAG density (RV3). At this point, the model has effectively computed an RV representing the acid density based on the propagation and functional combination of input random variables RV1, RV2, and RV3. It is evident that the derived acid count is not in fact an independent input RV but rather dependent on RV1, RV2, and RV3, respectively. 
Fig. 1

Depiction of MPPM model flow for the case of a chemically amplified resist.

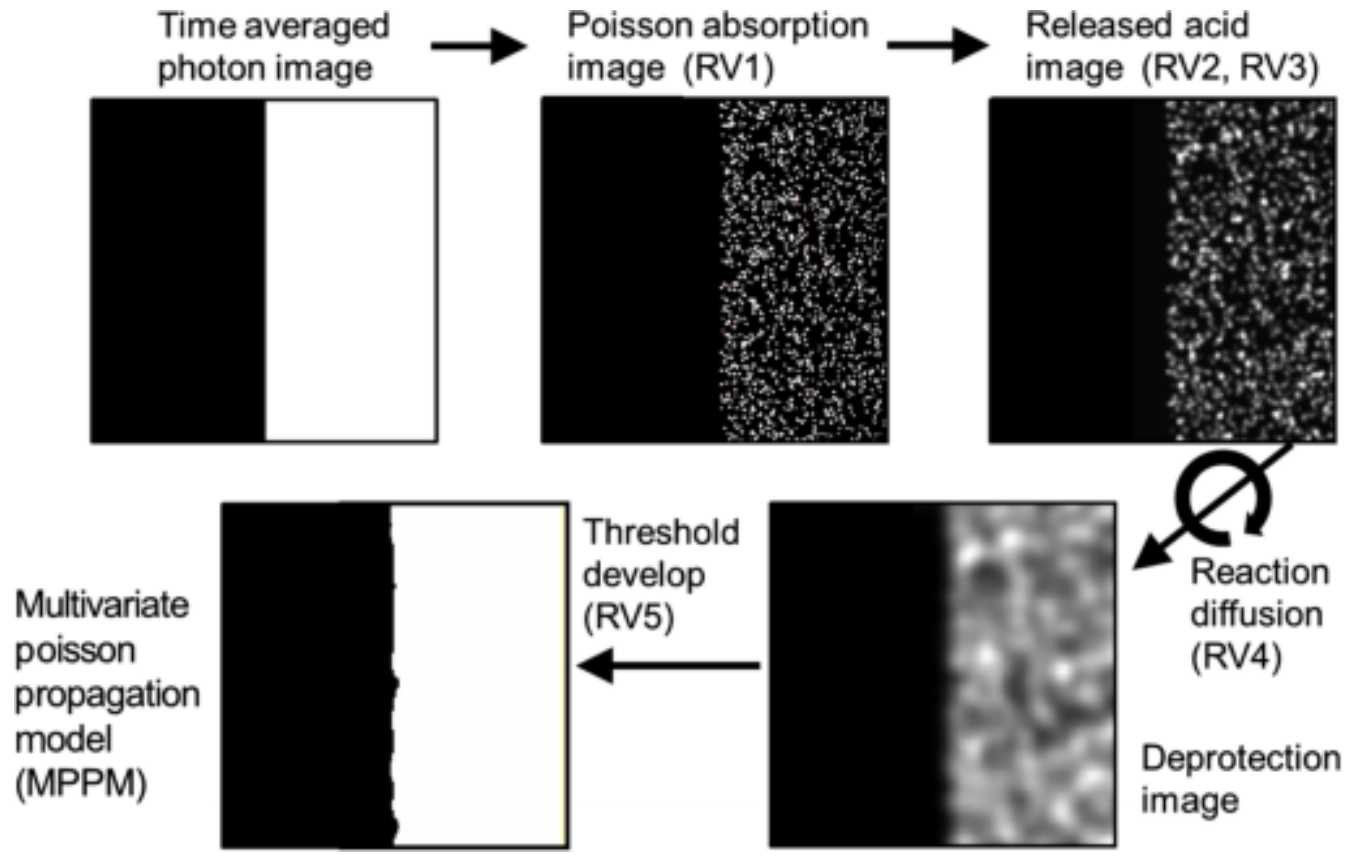

Now having computed the derived acid count RV, the next step in the model is the reaction diffusion process where the acid distribution reacts with the quencher (RV4) and the protecting groups (RV5).11 This is achieved through an iterative time-stepped process, where at each time step determinist acid and quencher blurs are applied, and simultaneous quenching and deprotection reactions are implemented based on deterministic reaction rates. The result of this process is to produce a single output RV that represents the deprotection ratio of the resist. Finally applying a develop model, which in the current implementation of the MPPM is simply a fractional threshold develop relative to $\mathrm{RV} 5$, we end up with the final output $\mathrm{RV}$ representing the dissolved fraction. We note that a more complicated stochastic dissolution model $\underline{12}, \underline{13}$ could also be applied. Table 1 shows a listing of the key input variables in the model noting whether they are stochastic or deterministic. All the stochastic terms are treated as Poisson.

Table 1

Listing of key input variables to the MPPM model.

Input parameter Type

Aerial image Deterministic

Absorptivity Deterministic

Absorbed photons Stochastic (RV1) 
Acid generation efficiency Stochastic (RV2)

Electron blur

PAG concentration

Quencher concentration

Protecting group concentration Stochastic (RV5)

Acid blur

Quencher blur

Acid/quencher reaction rate

Deprotection reaction rate

\section{Deterministic (Gaussian)}

Stochastic (RV3)

Stochastic (RV4)

Deterministic (Gaussian)

Deterministic (Gaussian)

Deterministic

Deterministic

Although described above from the perspective of a chemically amplified resist with conventional quencher, the model readily supports other modalities. For example, photo-decomposable quencher is implemented the same way as acid generation but in this case (RV2) represents the combined QE of the PAG activation and quencher decomposition, and a binomial distribution is used to allocate the events between PAG and quencher based on the local densities. In this case, the pre-reaction diffusion quencher count becomes a combination of the quencher distribution (RV4), the absorbed photon count (RV1), and the decomposition yield (which is determined by RV2). The model can also be applied to nonchemically amplified resists in which case RV3 becomes the density of photochemical-reactive sites in general instead of just PAG, quencher is removed, and the reaction diffusion equations are modified. The model can be summarized by stating that the various input RVs are propagated through the process and combined to yield the final output RV, which can be used to assess patterned variability. Depending on the input aerial image, the variability can take the form of line-edge roughness (LER), line-width roughness (LWR), contact CD uniformity, tip-to-tip variations. Also explicit in the model are a series of nonstochastic terms including absorptivity, diffusion lengths, and reaction rates. We note that these terms could also readily be converted to stochastic terms if desired.

3.

Limiting Behavior of Reduced Quencher Concentration

From the description above, it is evident that the final output RV of interest is basically a function of the input RVs (RV1 through RV5) and the MPPM can be thought of as a statistical error propagation problem. Also evident from the description above is that the acid is an intermediate derivative RV as compared with a fundamental input RV such as the photons, acid generation efficiency, PAG, and quencher and thus cannot be considered as an independent RV relative to the input terms. To understand the implications of this, it is instructive to analytically consider the intermediate acid noise $\mathrm{RV}$ and its relation to the input RVs. To render the analytic solution more tractable, we consider the simplified case where we ignore the PAG RV, ignore diffusion, and assume that the remaining RVs to be 
Gaussian with variance equal to the mean. Under this simplification, the acid RV (AA) can be expressed as

$A=\left[P \sum i=1 Y i\right]-Q, A=\left[\sum i=1 P Y i\right]-Q$,

where $\mathrm{PP}$ is the RV representing the absorbed photons, $\mathrm{YY}$ is the $\mathrm{RV}$ representing the acid generation efficiency, and $Q Q$ is the RV representing the quencher (nondecomposable). Now, the variance and mean of AAcan be written as

(2)

$\operatorname{Var}[\mathrm{A}]=\mathrm{E}[\mathrm{P}] \cdot \operatorname{Var}[\mathrm{Y}]+\operatorname{Var}[\mathrm{P}] \cdot \mathrm{E}[\mathrm{Y}] 2+\operatorname{Var}[\mathrm{Q}], \operatorname{Var}[\mathrm{A}]=\mathrm{E}[\mathrm{P}] \cdot \operatorname{Var}[\mathrm{Y}]+\operatorname{Var}[\mathrm{P}] \cdot \mathrm{E}[\mathrm{Y}] 2+\operatorname{Var}[\mathrm{Q}$ ],

(3)

$\mathrm{E}[\mathrm{A}]=\mathrm{E}[\mathrm{P}] \cdot \mathrm{E}[\mathrm{Y}]-\mathrm{E}[\mathrm{Q}], \mathrm{E}[\mathrm{A}]=\mathrm{E}[\mathrm{P}] \cdot \mathrm{E}[\mathrm{Y}]-\mathrm{E}[\mathrm{Q}]$, where $\operatorname{Var}[]$ represents the variance and $\mathrm{E}[] \mathrm{E}[]$ represents the expected value or mean. The fractional standard deviation of the acid (RNARNA) then becomes

(4)

$\mathrm{RNA}_{A}=\operatorname{sqrt}(\operatorname{Var}[\mathrm{A}]) / \mathrm{E}[\mathrm{A}]=\operatorname{sqrt}(\mathrm{E}[\mathrm{P}] \cdot \operatorname{Var}[\mathrm{Y}]+\operatorname{Var}[\mathrm{P}] \cdot \mathrm{E}[\mathrm{Y}] 2+\operatorname{Var}[\mathrm{Q}]) /(\mathrm{E}[\mathrm{P}] \cdot \mathrm{E}[\mathrm{Y}]-\mathrm{E}[\mathrm{Q}]) \cdot \mathrm{R}$ $\mathrm{NA}=\operatorname{sqrt}(\operatorname{Var}[\mathrm{A}]) / \mathrm{E}[\mathrm{A}]=\operatorname{sqrt}(\mathrm{E}[\mathrm{P}] \cdot \operatorname{Var}[\mathrm{Y}]+\operatorname{Var}[\mathrm{P}] \cdot \mathrm{E}[\mathrm{Y}] 2+\operatorname{Var}[\mathrm{Q}]) /(\mathrm{E}[\mathrm{P}] \cdot \mathrm{E}[\mathrm{Y}]-\mathrm{E}[\mathrm{Q}])$. Now we can consider what happens in the limit as the quencher count becomes very small both in absolute terms and relative to the absorbed photon count. In such a case, it is evident that the relative quencher noise would become very large, yet the relative acid noise does not blow up as the quencher effectively falls out of the equation:

(5)

$\mathrm{RNA}_{A}=\operatorname{sqrt}(\mathrm{E}[\mathrm{P}] \cdot \operatorname{Var}[\mathrm{Y}]+\operatorname{Var}[\mathrm{P}] \cdot \mathrm{E}[\mathrm{Y}] 2) /(\mathrm{E}[\mathrm{P}] \cdot \mathrm{E}[\mathrm{Y}]) \cdot \mathrm{RNA}=\operatorname{sqrt}(\mathrm{E}[\mathrm{P}] \cdot \operatorname{Var}[\mathrm{Y}]+\operatorname{Var}[\mathrm{P}] \cdot \mathrm{E}[\mathrm{Y}$ ] $2) /(E[P] \cdot E[Y])$.

This is not to say that quencher noise is inconsequential, it just means that in order for the quencher noise to play an important role it must be on par with the photon noise in absolute terms. We note that the singularity in Eq. (4) actually occurs when the mean quencher count is equal to the product of the mean absorbed photon count and mean acid yield. Under this limiting condition and this simplified view, all the produced acid is neutralized causing the denominator in Eq. (4) to go to zero.

The simplified analytic solution shown above is useful for the consideration of trends but not very useful for more quantitative analysis. Although the error propagation perspective does render a more general analytic solution plausible, a numerical implementation of the MPPM is certainly more convenient and flexible.

4.

Modeling Results

The modeling results described below were all obtained using a commercially available numeric implementation of the error propagation model.9 For the LER/LWR modeling, a 16-nm half-pitch aerial image is used [Fig. 2(a)]. The aerial image was calculated assuming a numerical aperture of 0.3 and dipole illumination 14 optimized for $32-\mathrm{nm}$ pitch. For the resist parameters we assume values typical of a chemically amplified resist

including: sensitivity $=33 \mathrm{~mJ} / \mathrm{cm} 2$ sensitivity $=33 \mathrm{~mJ} / \mathrm{cm} 2, Q E=2.8 Q E=2.8, P A G$ concentration $=0.2 / \mathrm{nm} 3 P A G$ concentration $=0.2 / \mathrm{nm} 3$, quenchercentration $=0.09 / \mathrm{nm} 3$ quenchercentration $=0.09 / \mathrm{nm} 3$, and protectinggroup concentration $=2 / \mathrm{nm} 3$ protectinggroup concentration $=2 / \mathrm{nm} 3$, absorptivity $=0.0042 / \mathrm{nmabsorptivity}=0.0042 / \mathrm{nm}$, thickness $=35 \mathrm{~nm}$ thickness $=35 \mathrm{n}$ 
$\mathrm{m}$, acid diffusion range $=10 \mathrm{nmacid}$ diffusion

range $=10 \mathrm{~nm}$, deprotectionrate $=1 \mathrm{~nm} 3 /$ sdeprotectionrate $=1 \mathrm{~nm} 3 / \mathrm{s}$, and quenching rate $=10 \mathrm{~nm} 3 / \mathrm{squenching}$ rate $=10 \mathrm{~nm} 3 / \mathrm{s}$. The quencher is assumed to be of the conventional (nonphotodecomposable) type. Figure 2(b) shows a resulting representative deprotection image.

Fig. 2

(a) 16-nm lines space input aerial image to the stochastic model and (b) stochastic model results for output deprotection function.
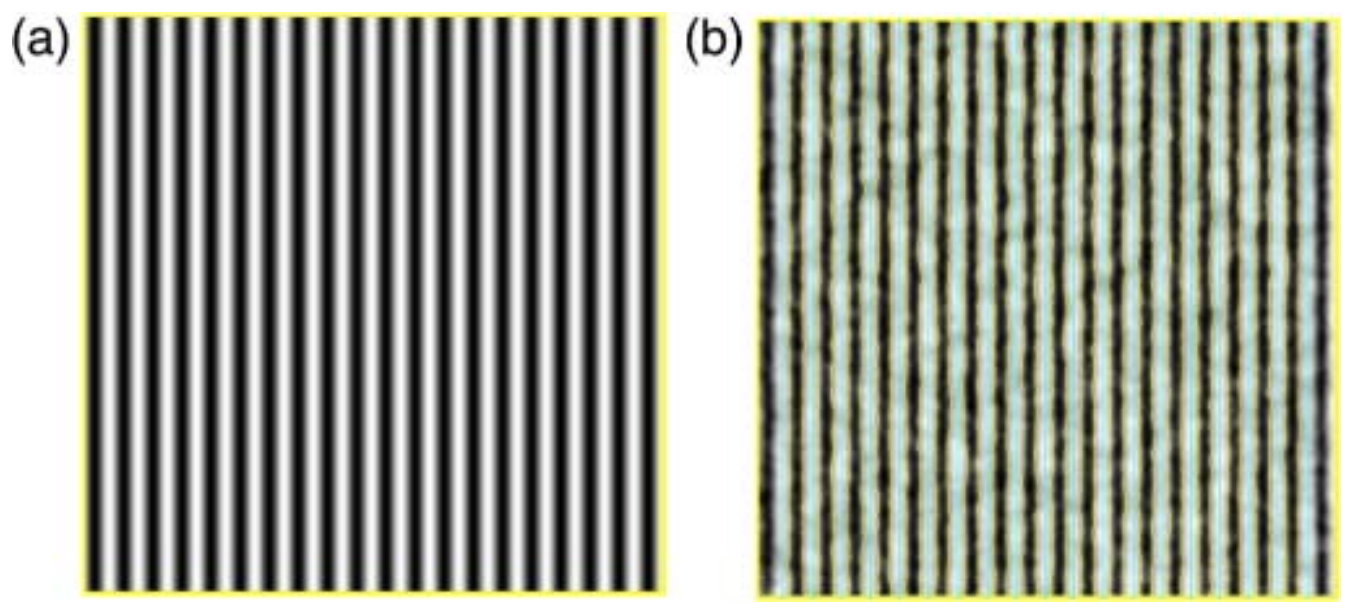

The dominant functions acting on the RVs in the propagation process are diffusion and reactiondiffusion processes that are essentially blur functions. Thus, as the stochastic terms propagate from the inputs to the output, spatial correlation is developed, which ultimately serves to band-limit the output noise (the LER/LWR). This can be observed through power spectral analysis of the modeled results. Figure 3 shows the measured low-frequency normalized LWR power spectral density (PSD) for three different acid diffusion range values: 8,12 , and $16 \mathrm{~nm}$. To characterize the PSD, a total of 180 lines were modeled with the individual line length being $640 \mathrm{~nm}$. PSD characterization was performed using a commercially available software package. 9 We show the low-frequency normalized PSD to visually highlight the impact of diffusion range on the correlation length. The impact on total roughness values (total area under the non-normalized PSD) is addressed below, where we show that although increasing diffusion range filters out higher spatial frequency roughness, the total roughness actually increases as a result of the total PSD magnitude increasing. The impact of the diffusion range is clearly evident in the PSD results, where we see the LWR correlation length (as nominally represented by the knee in the PSD) being proportional to the diffusion range.

Fig. 3

Calculated low-frequency-normalized LWR PSD from the stochastic model generated resist images. 


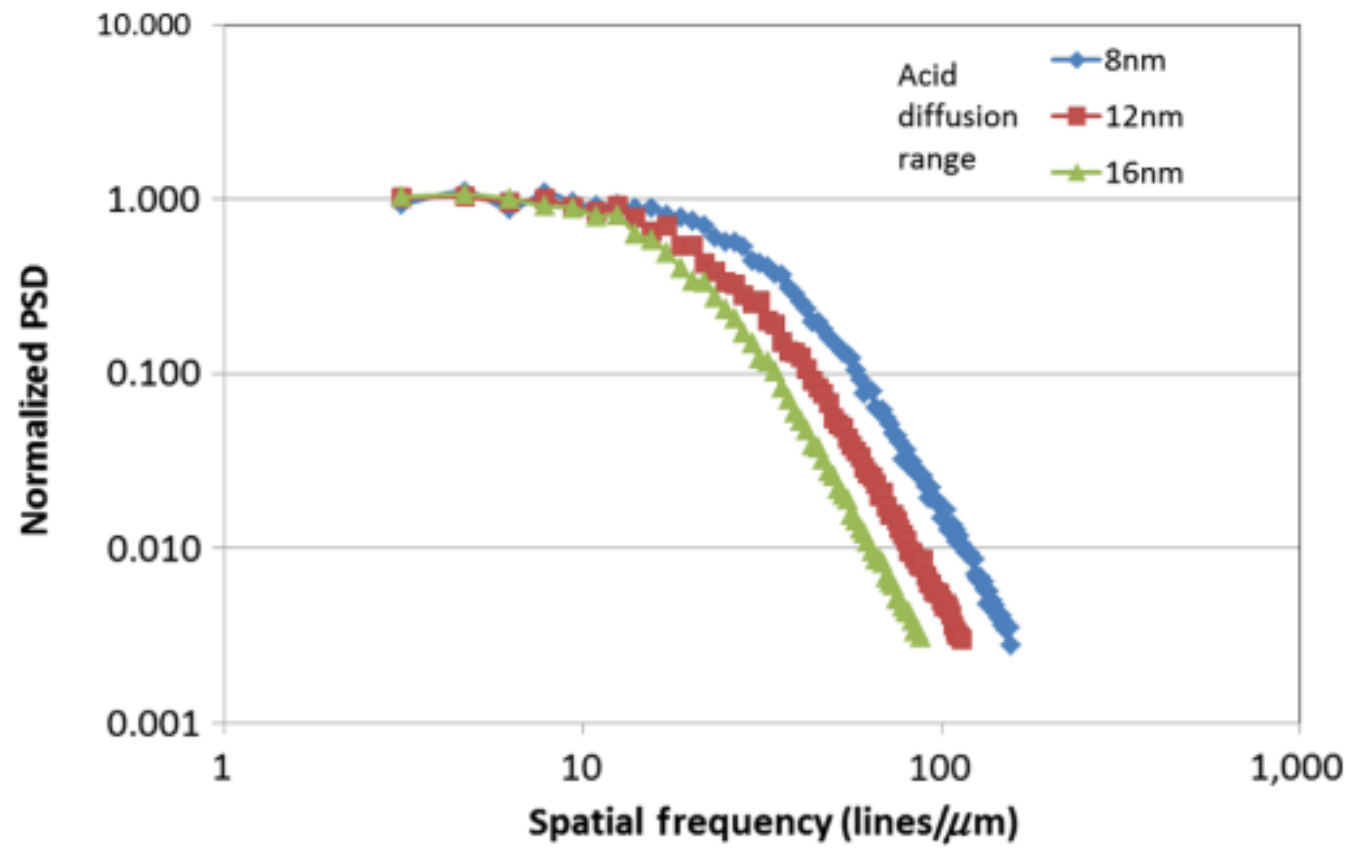

We note that the model-produced PSD shape is consistent with experimental data as is the dependence of the correlation length on blur.15 This lends credence to the model. Another well-established trend is LWR behavior as a function of quencher loading. To further demonstrate the validity of the model, we explicitly consider this case using the model parameters described above while varying only quencher loading and adjusting dose to maintain identical printed feature size. Figure 4 shows the results for modeled total LWR as a function of quencher concentration. The vertical error bars on the LWR arise from the size of the dataset used, 18 lines each 640-nm long (see Fig. 1). The modeled trends are consistent for both experimental results (for example, as shown in Ref. 16) as well as more complicated modeling results as shown in Ref. $\underline{6}$.

Fig. 4

Modeled LWR as a function of quencher concentration.

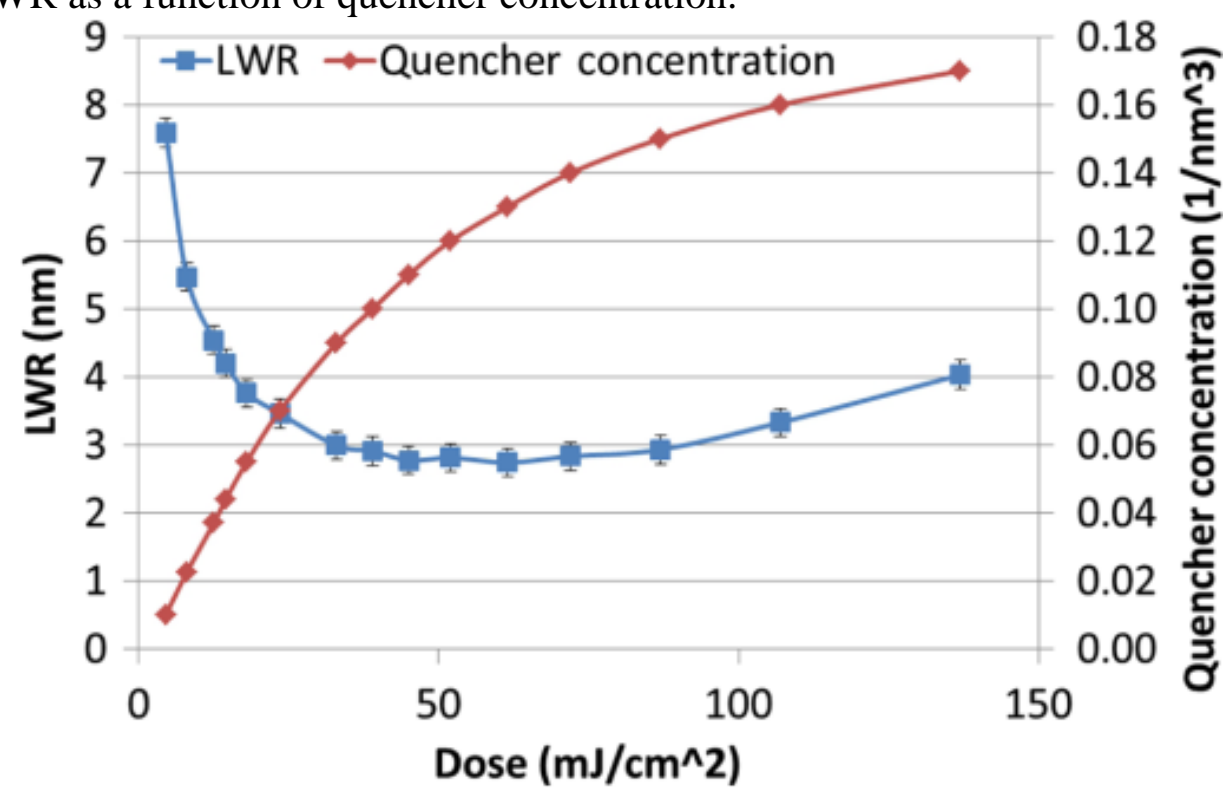


One of the benefits of this error propagation model is that isolating the effects of individual stochastic terms is very straightforward. Referring to Table 1, this can be achieved by setting all but one of the RVs to its corresponding mean value and running the model with the one remaining RV. For example, to isolate the effect of photon noise, RV2 through RV5 are replaced with their respective expected values, whereas RV1 is kept as stochastic. Table 2 shows the modeled LWR for the conditions described above considering each of the stochastic terms individually. We note that we could just as easily consider any combination of input stochastic terms. Because the individual stochastic terms are independent, the total stochastic impact is nominally the quadrature sum of the individual effects as opposed to the linear sum. In this case, the results show the photon noise to be the largest individual contributor but only slightly larger than the quencher effects. Note that when we consider the material terms only (disable the photon stochastics), we get a result of 2.3-nm LWR, which is larger than the photon alone effect, which is $1.9 \mathrm{~nm}$. Given that the LWR requirements for $16-\mathrm{nm}$ lines and spaces should be on the order of $1.2 \mathrm{~nm}$, we see that even for an infinitely slow resist (no photon noise), material constraints would still prevent us from meeting the requirement based primarily on the quencher noise. We note that the reported values have an uncertainty of $\sim \pm 2 \% \sim \pm 2 \% \mathrm{rms}$ based on the total amount of line length simulated relative to the LWR correlation length.

Table 2

Modeled LWR for 16-nm lines and spaces in a chemically amplified resist considering each of the stochastic terms individually and with all stochastic terms turned on simultaneously (bold).

\section{Stochastic terms $\quad$ LWR (nm)}

Photon (RV1 only) $\quad 1.9$

Acid generation efficiency (RV2 only) 1.2

PAG (RV3 only) $\quad 0.6$

Quencher (RV4 only) $\quad 1.9$

Protecting groups (RV5 only) $\quad 0.1$

\section{ALL (RV1 through RV5) $\quad 3.0$}

It is important to note that the results in Table 2 should not be interpreted as saying that the quencher noise is more important than acid noise. As described above, acid itself does not appear as an independent input noise term in this model, but rather it is a dependent-derived noise term. In fact, the quencher noise effectively imprints itself onto the derived acid noise (as do the photon and PAG noise terms), and thus the acid and quencher noise are dependent. This can also be seen in the simplified analytic error propagation expression shown in Eq. (2). In the MPPM model, acid noise is better viewed as a potential output RV of the error propagation process. That being said, deprotection is more representative of a resist image than is acid and thus is a more appropriate output RV to use for the evaluation of LWR.

Because the quencher appears as the dominant material term in Table 2, it is interesting to consider the dependence on quencher concentration. Figure 5 shows the model LWR breakdown for quencher 
concentration varying from 0.01 to $0.09 / \mathrm{nm} 30.09 / \mathrm{nm} 3$. To keep the dose fixed at $33 \mathrm{~mJ} / \mathrm{cm} 233 \mathrm{~mJ} / \mathrm{cm} 2$ across the dataset, we vary the deprotection rate from $1 \mathrm{~nm} 3 / \mathrm{s} 1 \mathrm{~nm} 3 / \mathrm{s}$ at the highest quencher concentration, down to $0.1 \mathrm{~nm} 3 / \mathrm{s} 0.1 \mathrm{~nm} 3 / \mathrm{s}$ at the lowest concentration. All other model parameters are held constant to isolate the impact of the quencher stochastics. The fact that acid diffusion range is independently defined as a model parameter means that we can consider quencher stochastics independent of the impact of quencher concentration on the diffusion range (diffusion range is separately considered below). Consistent with the analytic discussion above, the stochastic model shows the quencher-induced LWR to decrease even as the quencher count becomes very small (assuming one can simultaneously hold the diffusion range and dose constant). As described above, the deprotection rate was varied to keep the dose fixed, but we could have also kept the dose constant by varying, for example, QE and/or absorptivity. However, doing so would simultaneously be varying the RV1 and/or RV2 coupling in those trends that are separately explored later in the article.

Fig. 5

Model LWR breakdown as a function of quencher concentration. Deprotection rate (secondary axis) has been adjusted to keep dose constant.
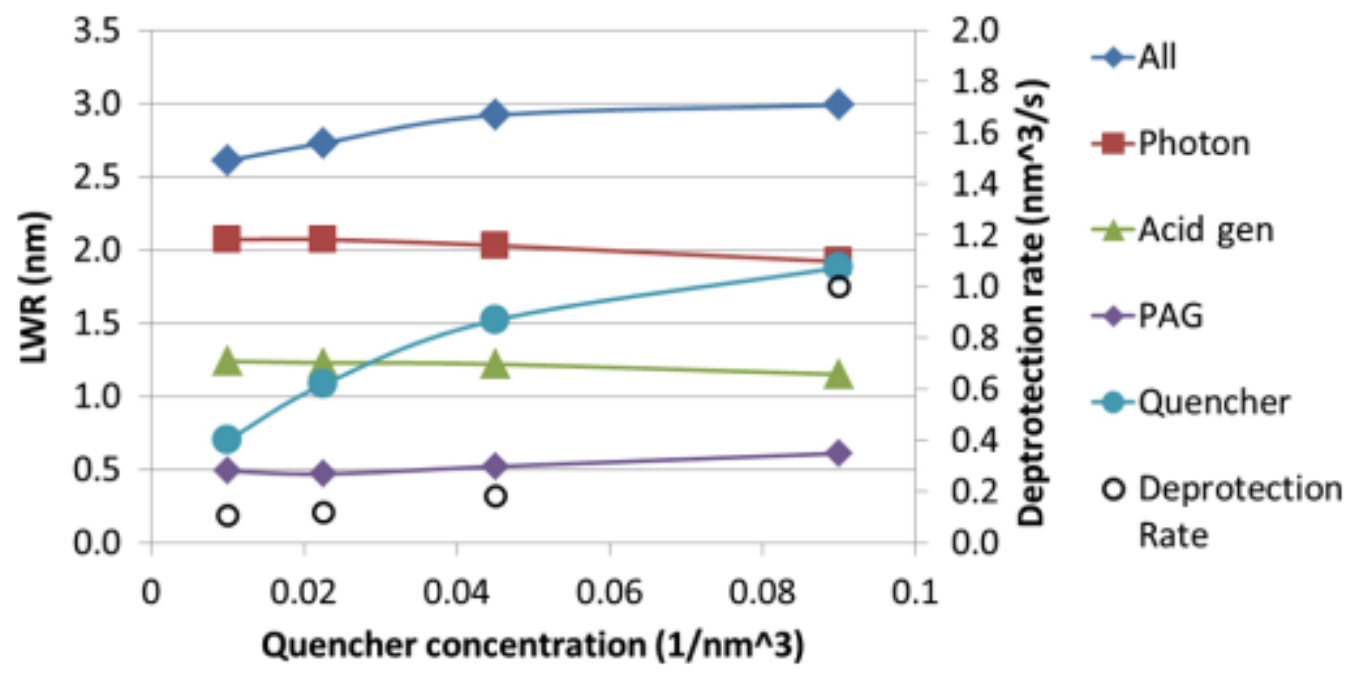

Next, we consider the case where the quencher is also photodecomposable. For this case, we assume the acid QE to remain the same and the quencher decomposition efficiency to be proportional to the acid QE based on the relative mean density of PAG and quencher. For example, if the mean quencher concentration is equal to the mean PAG concentration, then we assume the quencher decomposition efficiency to be equal to the acid QE. For the baseline photodecomposable quencher case to compare with Table 2, we set all resist parameters as described for Table 2 except for the quencher loading $(0.145 / \mathrm{nm} 30.145 / \mathrm{nm} 3)$, which is adjusted to achieve the same dose as for the nondecomposable case. Table 3 shows the results for the baseline case demonstrating a reduction in LWR compared with the nondecomposable case. The uncertainty is again $\pm 2 \% \pm 2 \% \mathrm{rms}$. The majority of the improvement comes from the quencher term that was reduced from $1.9 \mathrm{~nm}$ in Table 2 to $1.2 \mathrm{~nm}$ in Table 3. Nevertheless, the quencher remains the dominant material term. Figure 6 shows the dependence on quencher concentration for the photodecomposable quencher case. The deprotection rate is again adjusted to keep the dose fixed, varying from $0.1 \mathrm{~nm} 3 / \mathrm{s} 0.1 \mathrm{~nm} 3 / \mathrm{s}$ at a quencher concentration of $0.01 / \mathrm{nm} 30.01 / \mathrm{nm} 3$ to $3.5 \mathrm{~nm} 3 / \mathrm{s} 3.5 \mathrm{~nm} 3 / \mathrm{s}$ at a quencher concentration of $0.2 / \mathrm{nm} 30.2 / \mathrm{nm} 3$. In this case, we find a nonzero optimum for quencher concentration. It is assumed that this behavior is a result of the trade-off between the reduced noise as discussed above and the chemical slope dependence on 
photodecomposable quencher concentration. An increase in the photon-induced LWR at the high quencher concentration is a result of the degradation of chemical slope. As one would expect, at very low concentrations, the system reverts to the behavior seen for the conventional quencher case where the quencher term becomes less important both in terms of both noise and slope.

Table 3

Modeled LWR for 16-nm lines and spaces in a chemically amplified resist with photo-decomposable quencher considering each of the stochastic terms individually and with all stochastic terms turned on simultaneously (bold).

\section{Stochastic terms $\quad$ LWR (nm)}

Photon 1.9

Acid generation efficiency 0.9
PAG

Quencher

1.2

Protecting groups

0.1
ALL
2.7

Fig. 6

Model LWR breakdown as a function of quencher concentration for photodecomposable quencher case. Deprotection rate (secondary axis) has been adjusted to keep dose constant.
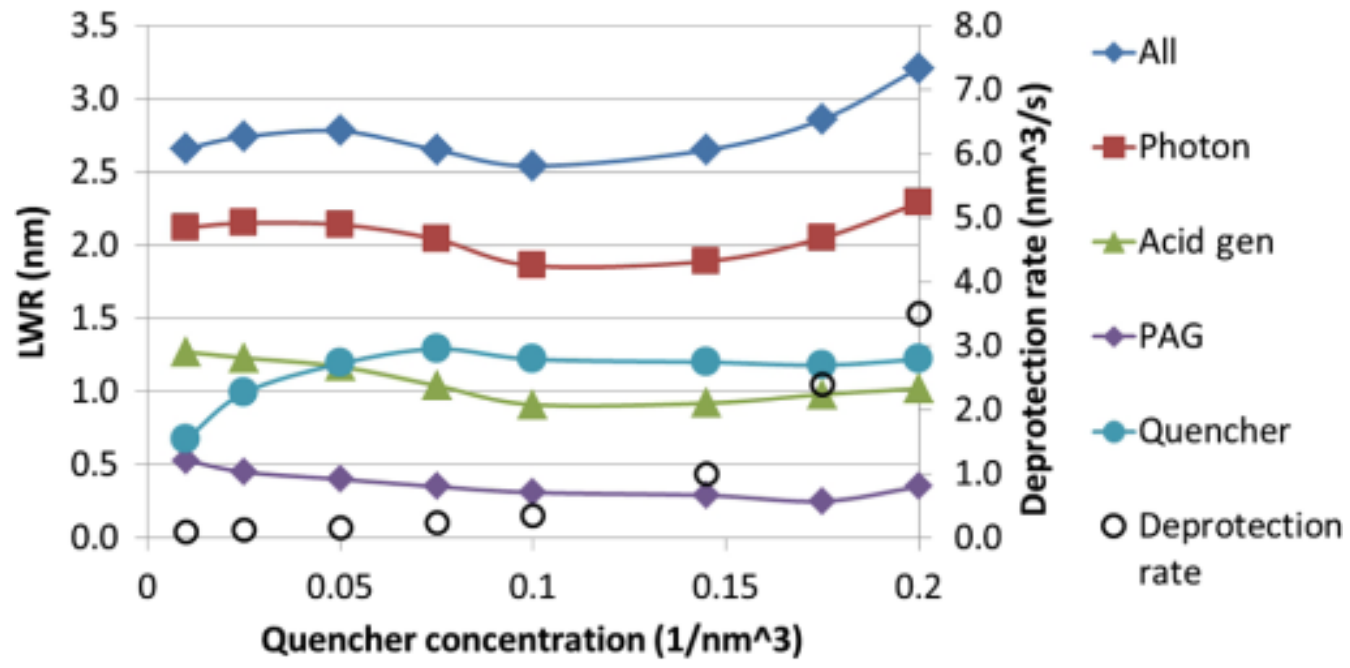

Next, we consider the dependence on QE (acid generation efficiency, RV2) as shown in Fig. 7. We explore both photodecomposable quencher (density of $0.145 / \mathrm{nm} 30.145 / \mathrm{nm} 3$ ) and conventional quencher (density of $0.09 / \mathrm{nm} 30.09 / \mathrm{nm} 3$ ) cases and again simultaneously adjust the deprotection rate to ensure that the dose remains fixed. In general, we see the LWR to improve as the QE is increased. The 
dominant reasons for this improvement are the reduction in the relative stochastics of the QE value itself and the fact that mean acid count is growing relative to the mean quencher count thus reducing the impact of the quencher stochastics. This is again consistent with the simplified analytic view shown in Eq. (4).

Fig. 7

Model LWR breakdown as a function of QE for (a) conventional quencher and (b) photodecomposable quencher. Deprotection rate (secondary axis) has been adjusted to keep dose constant.
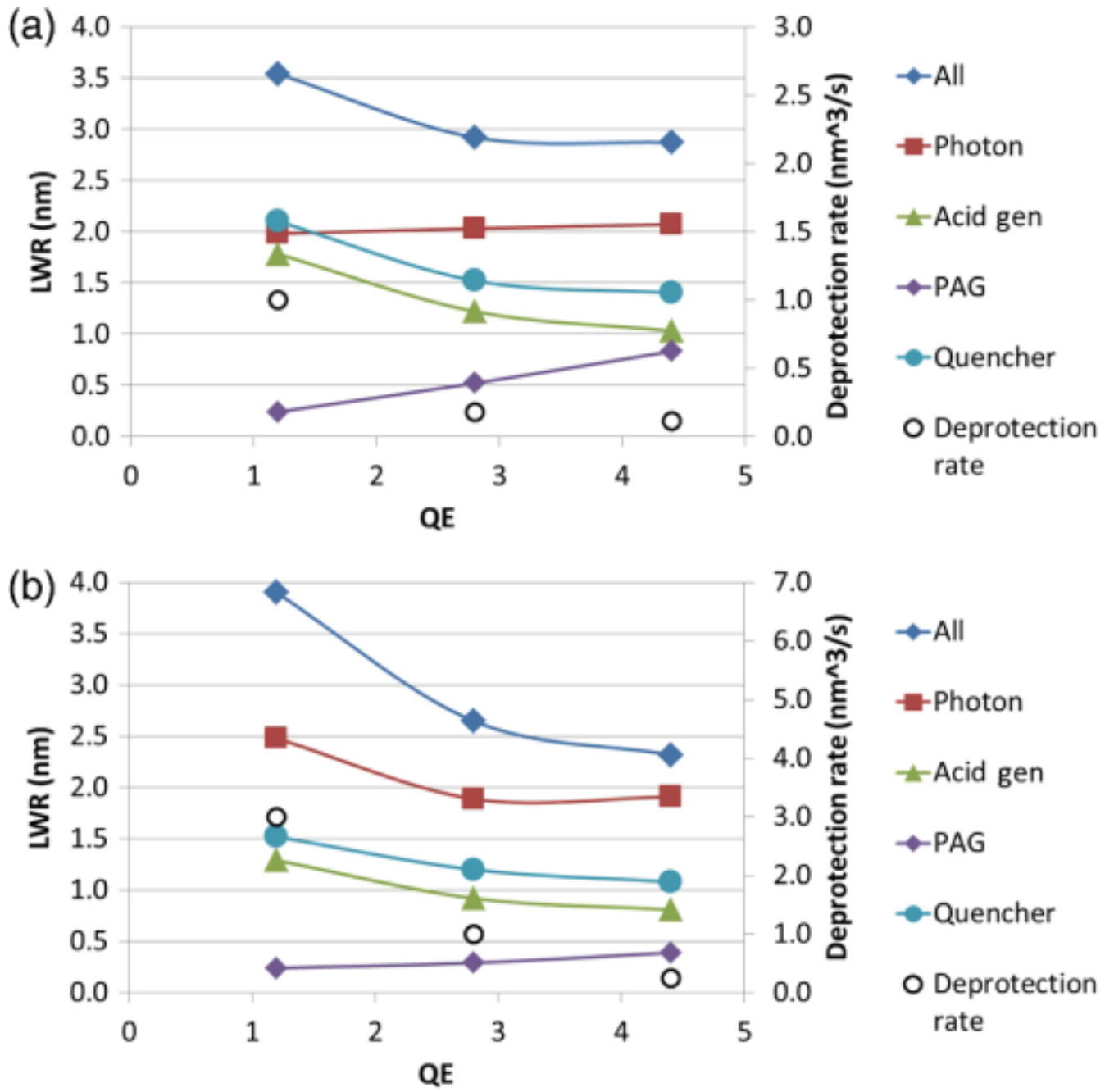

Although the impact of acid diffusion range on the low-frequency normalized PSD was explored in Fig. 3 , Fig. 8 shows the impact of acid diffusion range on the total roughness and individual component magnitudes for the conventional quencher case described above (quencher concentration $=0.09 / \mathrm{nm} 3$ quencher concentration $=0.09 / \mathrm{nm} 3$ ). The results demonstrate that there is in fact an optimal diffusion range in terms of the LWR. This finding is fully consistent with the heuristic view of the trade-offs involved: increase diffusion range serves to filter out noise that reduces LWR, but taken too far, the loss of chemical image slope begins to dominate, which effectively represents the susceptibility to noise. Figure 9 shows the power spectra from Fig. 3 but now redisplayed without normalization. At below optimal blur, the total roughness increase comes from the high-frequency components, whereas at higher than optimal blur the total roughness increase comes from an increase 
in the magnitude of the low-frequency components. Identical trends (not shown here) as a function of acid diffusion range are observed for the photodecomposable quencher case.

Fig. 8

Model LWR breakdown as a function of acid diffusion range.

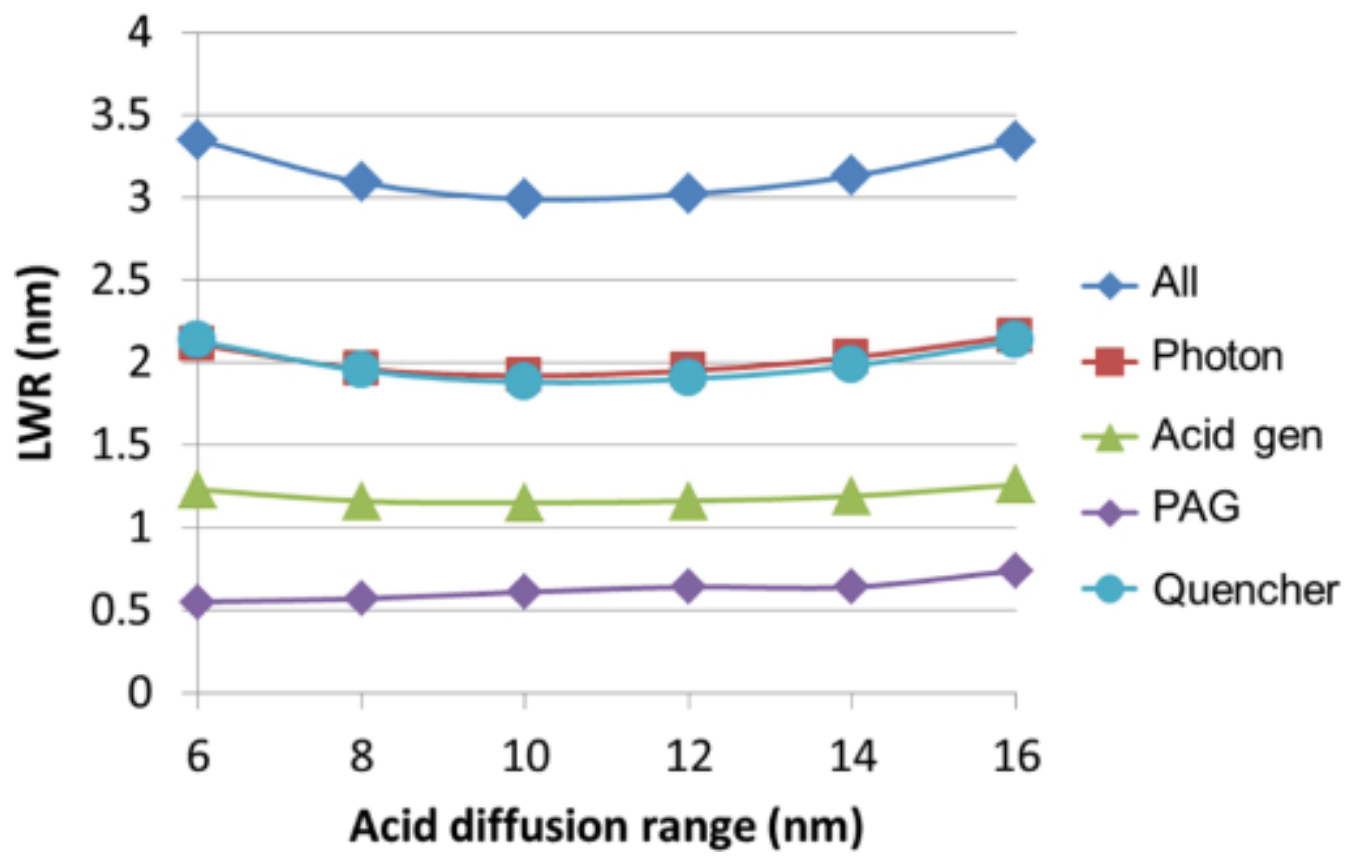

Fig. 9

LWR PSD from Fig. 3 without normalization.

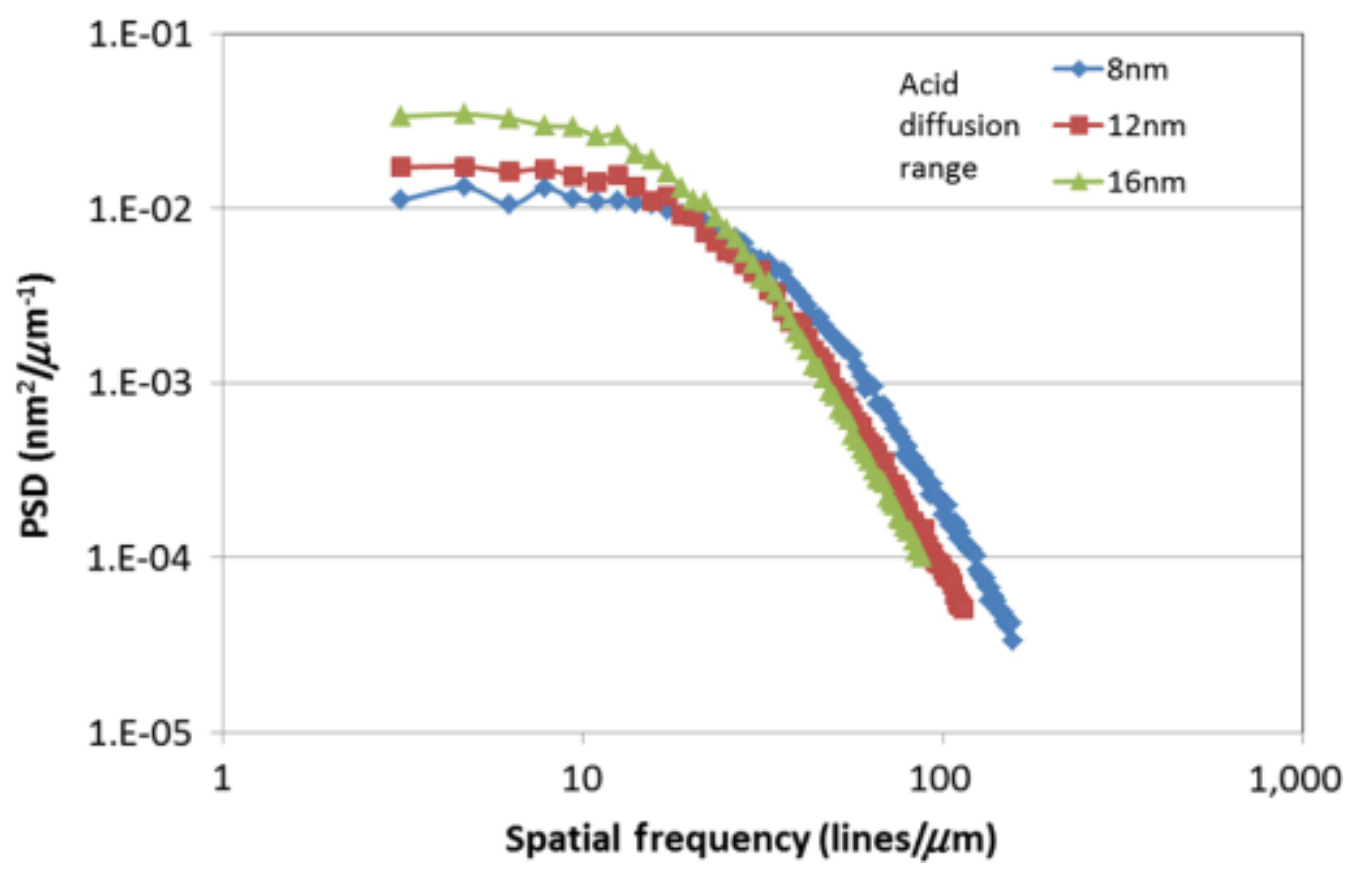


An arguably critical term not addressed above is the absorptivity. From the model perspective, however, the impact of this term is readily understood analytically. Because the process starts based on the number of photons absorbed, increasing absorptivity can be shown to impact nothing but the required dose. For example, in all the cases above, the dose was $33 \mathrm{~mJ} / \mathrm{cm} 233 \mathrm{~mJ} / \mathrm{cm} 2$ and the absorptivity was $0.0042 / \mathrm{nm} 0.0042 / \mathrm{nm}$; should we increase the absorptivity to $0.0084 / \mathrm{nm} 0.0084 / \mathrm{nm}$, the only change would be that the required dose would drop to $16.5 \mathrm{~mJ} / \mathrm{cm} 216.5 \mathrm{~mJ} / \mathrm{cm} 2$ and all the plots presented above would remain identical. Clearly, absorptivity is a powerful term and arguably can be seen as bypassing the conventional trade-offs in that it can be used to increase photospeed with no negative impact on LWR and resolution.

The conclusion above, however, depends on the ability to increase absorptivity without impacting any of the other resist parameters including acid generation efficiency and reaction rates. If on the other hand, absorptivity is increased but the QE decreases such that the dose remains fixed, the benefits are much less evident as shown in Fig. 10. To the extent the system is photon limited, LWR improvement is achieved, but the gains are constrained by the limiting material terms that are not impacted by absorptivity. Should the resist start off in the material limited condition, absorptivity increase would provide no benefit in LWR.

\section{Fig. 10}

Model LWR breakdown as a function of absorptivity. QE (secondary axis) has been adjusted to keep dose constant.

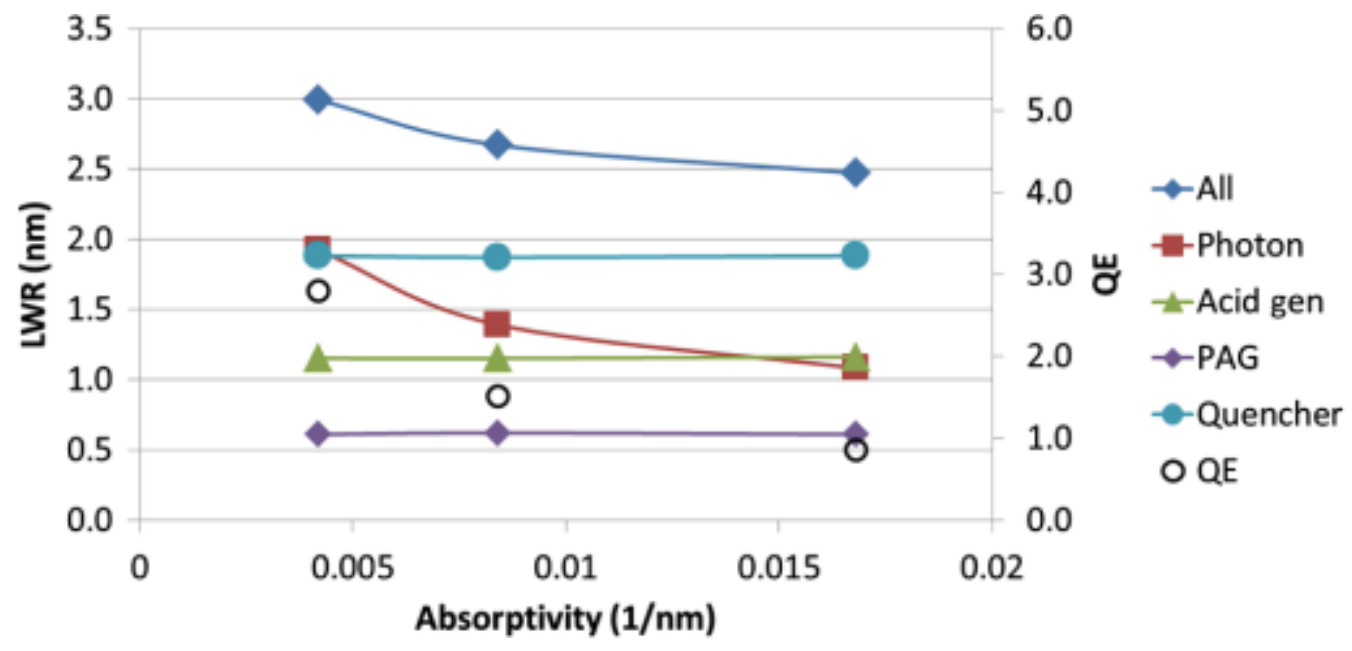

Alternatively, if the absorptivity is increased at a fixed QE, but the PAG concentration is increased proportionally while also decreasing the deprotection rate to keep the dose fixed, the benefits on LWR become much more significant (Fig. 11). In this case, both the photon and material terms benefit. In Fig. 11, the deprotection rate is decreased from an initial value of 1 to $0.045 \mathrm{~nm} 3 / \mathrm{s} 0.045 \mathrm{~nm} / \mathrm{s}$ as the absorptivity is increased from 0.0042 to $0.0168 / \mathrm{nm} 0.0168 / \mathrm{nm}$. To benefit from increased absorptivity, one must also maintain chemical yields.

\section{Fig. 11}

Model LWR breakdown as a function of absorptivity with fixed QE, proportionally increasing PAG concentration and decreasing deprotection rate (secondary axis) to keep dose constant. 


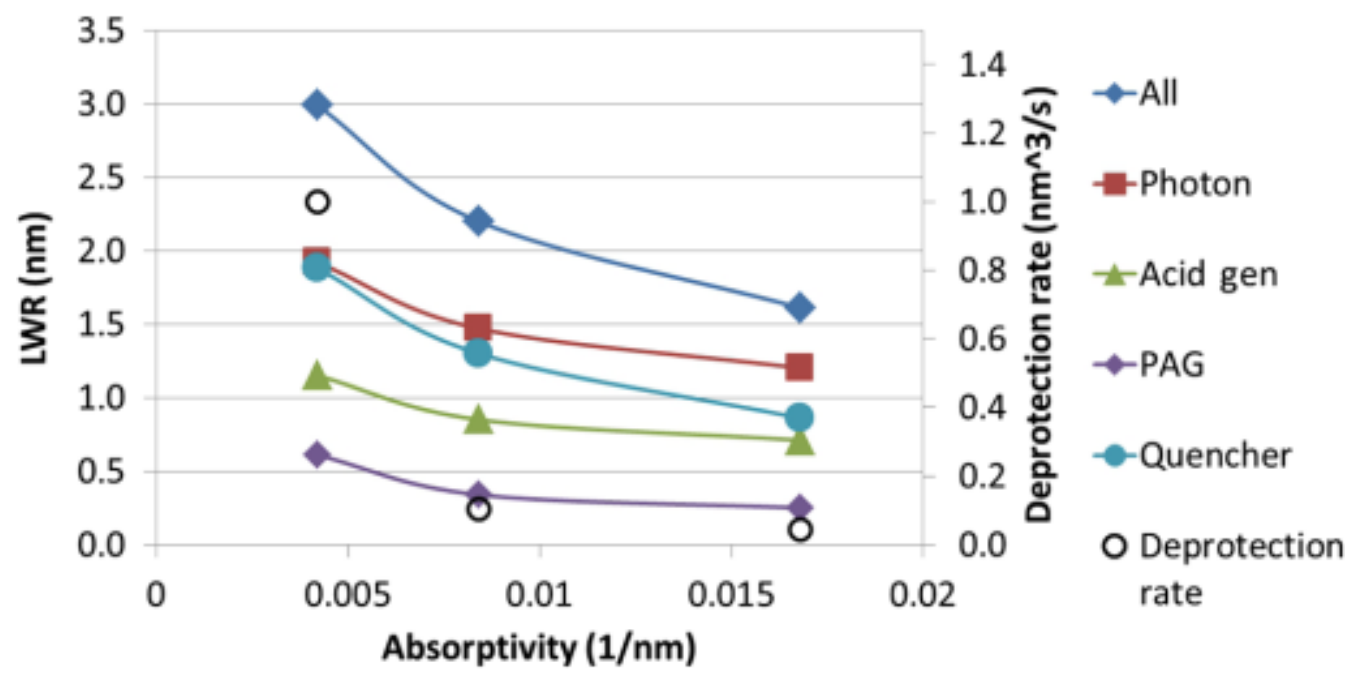

5.

Conclusion

An error propagation stochastic model has been described and used to study the impact of both photon and photoresist material sources of LWR. Based on typical chemically amplified resist parameters, material sources of LWR are shown to be of equal importance to photon sources. Of the material sources, quencher is shown to be the most important input noise term. It is important to note that it is not the relative quencher noise that ultimately matters but rather the absolute quencher noise relative to the mean acid count. Assuming the deprotection blur and sensitivity could be controlled, the stochastic modeling shows that a simpler resist with no quencher would be preferable; however, it is not clear how this could be achieved in practice with a conventional chemically amplified resist. The model has also been used to study the impacts of photodecomposable quencher, QE, and acid blur as well as the behavior of the LWR PSD. The inclusion of photodecomposable quencher allows the negative impacts of additional quencher noise to be offset by an improvement in chemical slope, yielding a nonzero optimum for quencher concentration. Finally, the benefit of increased absorptivity was also shown as was the importance of coupling the increase to maintained chemical yields.

Acknowledgments

This work was performed at Lawrence Berkeley National Laboratory with support from Intel, Samsung, EUV Tech, Inpria, and JSR through the U.S. Department of Energy under Contract No. DE-AC02$05 \mathrm{CH} 11231$. 